\title{
Electron Microscopy Study of Preferential Variant Selection in CoPt Alloy Ordered under a Magnetic Field
}

\author{
Hiroshi Akamine ${ }^{1}$, Sahar Farjami ${ }^{2, *}$, Masatoshi Mitsuhara ${ }^{2}$, Minoru Nishida ${ }^{2}$, \\ Takashi Fukuda ${ }^{3}$ and Tomoyuki Kakeshita ${ }^{3}$ \\ ${ }^{1}$ Department of Applied Science for Electronics and Materials, Interdisciplinary Graduate School of Science and Engineering, \\ Kyushu University, Kasuga 816-8580, Japan \\ ${ }^{2}$ Department of Engineering Science for Electronics and Materials, Kyushu University, Kasuga 816-8580, Japan \\ ${ }^{3}$ Department of Materials Science and Engineering, Graduate School of Engineering, Osaka University, Suita 565-0871, Japan
}

Transmission electron microscope (TEM) and scanning transmission electron microscope (STEM) observations were carried out to investigate microstructure formation and variant selection process in $\mathrm{L}_{0}$-type ordered CoPt alloy upon a two-step ordering heat-treatment. The first step corresponds to nucleation process carried out under a magnetic field of $10 \mathrm{~T}$ and the second step represents growth process without magnetic field. After the first step of ordering, ordered domains of about $5 \mathrm{~nm}$ in size were observed and fraction of the preferred variant with the $c$-axis parallel to applied magnetic field was slightly higher than that of the other two variants. Formation of tweed microstructure along $\{011\}_{\mathrm{L} 1_{0}}$ was confirmed at the initial stage of ordering. This structure is considered to be derived from the periodic alignment of interface between two ordered variants with twin relation. At the early stage of the second step of ordering, numerous micro-twins were formed through tweed microstructure and the volume fraction of the preferred variant was increased accompanying with modulation of twins, while that of other two variants was decreased. After the second step of ordering, the twins were vanished and single variant was obtained.

[doi:10.2320/matertrans.MAW201313]

(Received April 25, 2013; Accepted June 17, 2013; Published August 25, 2013)

Keywords: CoPt alloy, transmission electron microscopy (TEM), scanning transmission electron microscopy (STEM), high-angle annular dark field (HAADF), magnetic field, disorder-order transformation, tweed microstructure

\section{Introduction}

It is well known that there are three variants of $\mathrm{L} 1_{0}$-type ordered phase originated from the tetragonal symmetry in near-equiatomic $\mathrm{Co}-\mathrm{Pt}$, Fe-Pd and Fe-Pt alloys. This multivariant structure prevents to exhibit the intrinsic high magnetic anisotropy in these alloys. Therefore, it is inevitable to improve the magnetic anisotropy by controlling formation and rearrangement of variants. Many efforts have been done in this regard and a promising method is known to be application of external fields during the ordering process. ${ }^{1-6)}$ Magnetic field is one of such external fields expected to enhance variant selection in $\mathrm{Co}-\mathrm{Pt}$, $\mathrm{Fe}-\mathrm{Pd}$ and $\mathrm{Fe}-\mathrm{Pt}$, since the ordered phase of these alloys is ferromagnetic with high uniaxial magnetocrystalline anisotropy. In fact, single variant state has been achieved by a two-step ordering heat-treatment under a magnetic field in $\mathrm{Co}-\mathrm{Pt}^{5)}$ and $\mathrm{Fe}-\mathrm{Pd} .{ }^{6)}$ The results suggest that formation of multi or single variant state is controlled in the first step of ordering under a magnetic field. However, the process of single variant formation is not well understood from the viewpoint of microstructure. Especially, there have been only a few reports on microstructure observation in $\mathrm{Co}-\mathrm{Pt}$ alloy compared to that in $\mathrm{Fe}-\mathrm{Pd}$ alloy because of difficulty in specimen preparation due to the inert nature of Pt.

In the present study, therefore, ordering process and variant selection during the two-step ordering heat-treatment have been investigated in $\mathrm{CoPt}$ alloy by transmission electron microscopy (TEM) and scanning transmission electron microscopy (STEM).

${ }^{*}$ Corresponding author, E-mail: farjami@asem.kyushu-u.ac.jp

\section{Experimental Procedure}

An ingot of CoPt $(\mathrm{Co}-50$ at\% $\mathrm{Pt})$ was prepared by arc melting using degassed electrolytic cobalt and platinum plate as starting materials. A single crystalline rod was grown by a floating zone method and homogenized at $1273 \mathrm{~K}$ for $168 \mathrm{~h}$ in an evacuated quartz tube $\left(2.0 \times 10^{-4} \mathrm{~Pa}\right)$ followed by quenching into ice water. After determining the crystallographic orientation by Laue method, some cubic specimens with $\{100\}_{\mathrm{A} 1}$ were cut out. Each edge of the specimen is about $2.0 \mathrm{~mm}$. In this paper, three, $[100]_{\mathrm{A} 1},[010]_{\mathrm{A} 1}$ and $[001]_{\mathrm{A} 1}$ axes are defined as $\mathrm{X}-$, Y- and Z-directions, respectively. The Curie temperature of the disordered and ordered phases was 850 and $730 \mathrm{~K}$, respectively, measured by electrical resistivity. ${ }^{5,6}$ These cubic specimens were heattreated at $1273 \mathrm{~K}$, which is above the disorder-order transformation temperature of $1045 \mathrm{~K},{ }^{5)}$ and then quenched into ice water. The quartz tube was crashed immediately to suppress the ordering. The following lattice parameters determined by X-ray diffraction experiments were used in the analysis of selected area electron diffraction pattern (SAEDP) obtained from the disordered A1 and the ordered $\mathrm{L1}_{0}$ phases: $a_{0}=0.377$, and $a=0.381$ and $c=0.371 \mathrm{~nm}$ $(c / a=0.974)$, respectively.

The two-step ordering heat-treatment diagram is schematically illustrated in Fig. 1. The first and second steps correspond to nucleation and growth processes, respectively. In order to investigate various stages of the growth process, appropriate heat-treatment conditions were selected as listed in Table 1. Specimens for TEM and STEM observations were prepared as follows. Square plates parallel to the $[001]_{\mathrm{A} 1}$ axis were cut out from the cubic specimens and then mechanically polished to about $100 \mu \mathrm{m}$ in thickness. Because 


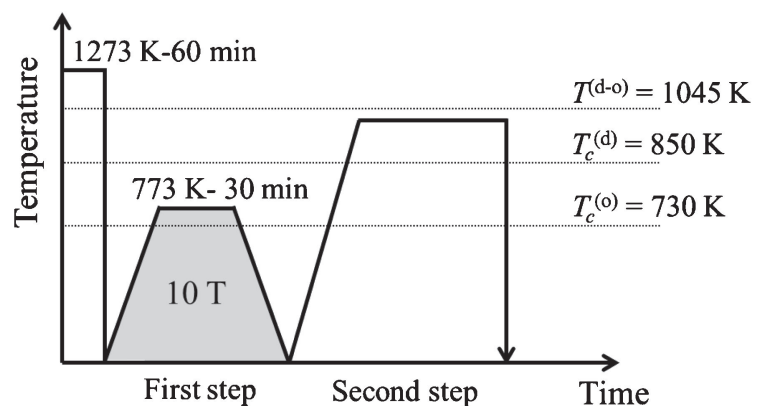

Fig. 1 Schematic diagram of the two-step ordering heat-treatment. Heattreatment conditions of the second step are summarized in Table 1.

Table 1 Conditions of the two-step ordering heat-treatment.

\begin{tabular}{ccc}
\hline Specimen & First step & Second step \\
\hline A & $773 \mathrm{~K}, 30 \mathrm{~min}, 10 \mathrm{~T}$ & \\
B & $773 \mathrm{~K}, 30 \mathrm{~min}, 10 \mathrm{~T}$ & $773 \mathrm{~K}, 20 \mathrm{~min}$ \\
C & $773 \mathrm{~K}, 30 \mathrm{~min}, 10 \mathrm{~T}$ & $873 \mathrm{~K}, 90 \mathrm{~min}$ \\
D & $773 \mathrm{~K}, 30 \mathrm{~min}, 10 \mathrm{~T}$ & $1023 \mathrm{~K}, 3 \mathrm{~min}$ \\
\hline
\end{tabular}

of inert nature of Pt-based alloys as mentioned above, it is difficult to prepare the specimens by common electrochemical manner. They were dimpled and finally finished by argon ion milling. TEM and STEM observations were carried out by JEM-2000EX and JEM-ARM-200F operated at $200 \mathrm{kV}$, respectively.

\section{Results and Discussions}

Figure 2(a) shows the SAEDP obtained from the specimen A which is heat-treated at $773 \mathrm{~K}$ for $30 \mathrm{~min}$ under a magnetic field of $10 \mathrm{~T}$ as listed in Table 1. The pattern consists of three sets of reflections derived from X-, Y- and
Z-variants of $\mathrm{L}_{0}$ ordered phase as indicated by key diagrams in Figs. 2(b), 2(c) and 2(d), respectively. Direction of applied magnetic field is indicated by the vertical arrow $H$ in Fig. 2. Although it is difficult to adjust the exact Bragg condition due to the ferromagnetic nature of the specimen, the intensity of $001_{\mathrm{Z}}$ superlattice reflection (SLR) is obviously stronger than that of $110_{\mathrm{X}}$ and $001_{\mathrm{Y}}$ SLRs. In particular, it can be concluded that there is significant difference of the intensity between $001_{\mathrm{Z}}$ and $001_{\mathrm{Y}}$ SLRs, since both SLRs have the same distance from the transmitted electron beam. Figures 2(e), 2(f) and 2(g) are dark field images (DFIs) taken by using $110_{\mathrm{X}}, 001_{\mathrm{Y}}$ and $001_{\mathrm{Z}}$ SLRs, respectively. It is apparent that the bright dot contrast in each of DFIs corresponds to the ordered $\mathrm{L}_{0}$ phase. The density of bright dots in ( $g$ ) is higher than that in (e) and (f) as expected from the intensity of each SLR in (a). These observations suggest that the preferential nucleation of the Z-variant occurs at the first step of ordering. These results are consistent with the magnetization measurement. ${ }^{5,7)}$ At this stage, tweed microstructure with striations along $\langle 011\rangle_{\mathrm{L} 1_{0}}$ directions is clearly observed especially in DFIs of the Y- and Z-variants in Figs. 2(f) and 2(g). Weak elongation and/or split observed in the SLRs and fundamental reflections in Fig. 2(a) are considered to be mainly due to the tweed microstructure. In addition, it should be taken into account the superimposition of reflections derived from $\mathrm{X}$-, Y- and Z-variants and the effect of $c / a$ ratio. However, it is difficult to separate these factors at present. It is well recognized that the tweed microstructure is frequently observed as a microstructural aspects of pre-martensitic phenomena ${ }^{8)}$ or the initial stage of disorder-order transformation from a cubic to tetragonal phase. ${ }^{9,10)}$ The reason for formation of the tweed microstructure is attributed to the local tetragonal distortion in the cubic structure. ${ }^{11)}$ Figure 3(a) is a high-angle annular dark field (HAADF)-STEM image of the tweed microstructure in specimen A. Figures 3(b) and 3(c) are enlarged micrographs

(a) (b)

(c)

(d)

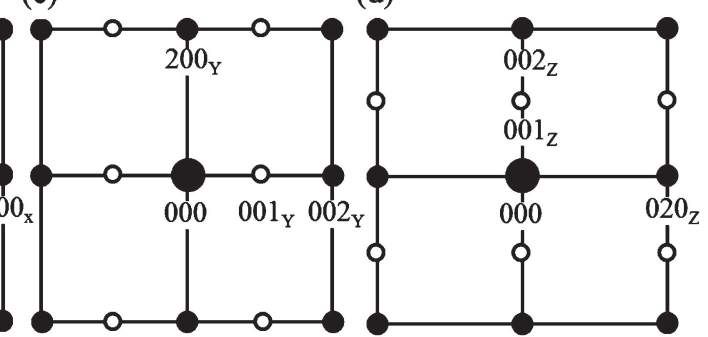

$\mathrm{EB} / /[010]_{\mathrm{Y}}$

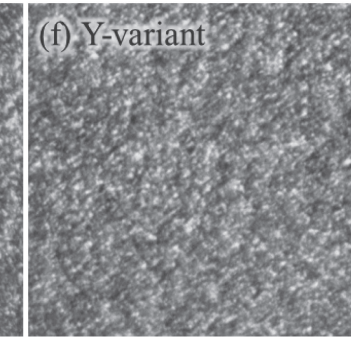

$\mathrm{EB} / /[100]_{Z}$

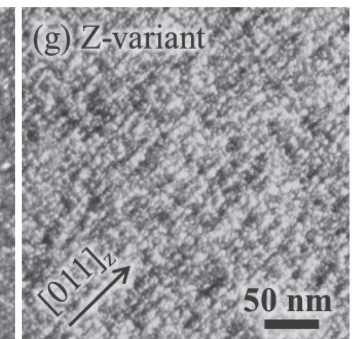

Fig. 2 (a) SAEDP for specimen A heat-treated at $773 \mathrm{~K}$ for $30 \mathrm{~min}$ under a magnetic field of $10 \mathrm{~T}$ in the first step of ordering. (b)-(d) Schematic key diagram of diffraction patterns derived from X-, Y- and Z-variants. In key diagrams of (b)-(d), black and white circles represent fundamental and superlattice reflections, respectively. (e)-(g) DFIs of X-, Y- and Z-variants taken by $u$ sing $110_{X}, 001_{Y}$ and $001_{Z}$ SLRs in (a). 


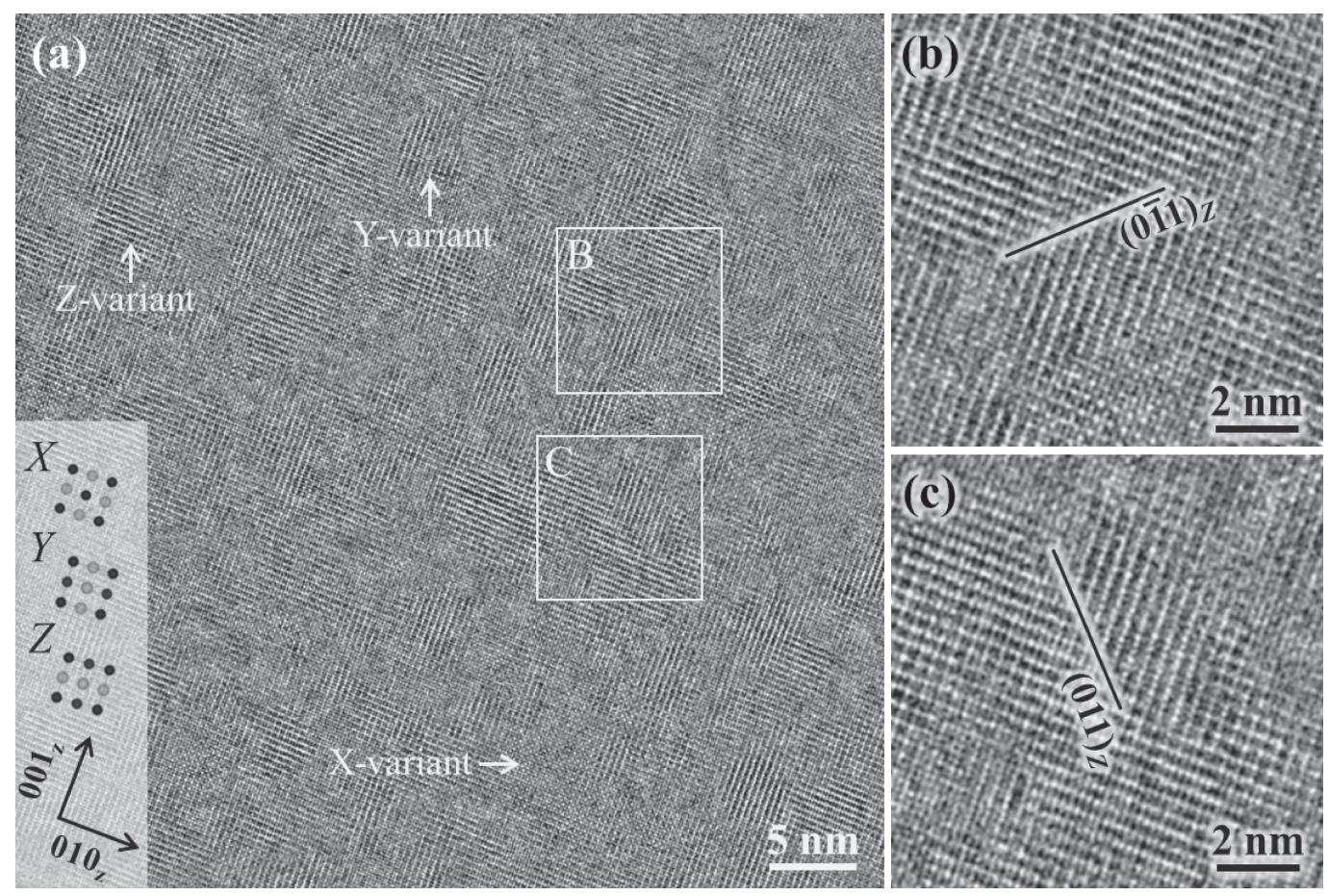

Fig. 3 (a) HAADF-STEM image of specimen A heat-treated at $773 \mathrm{~K}$ for $30 \mathrm{~min}$ under a magnetic field of $10 \mathrm{~T}$ in the first step of ordering. (b) and (c) Enlarged images of areas B and C in (a), respectively.
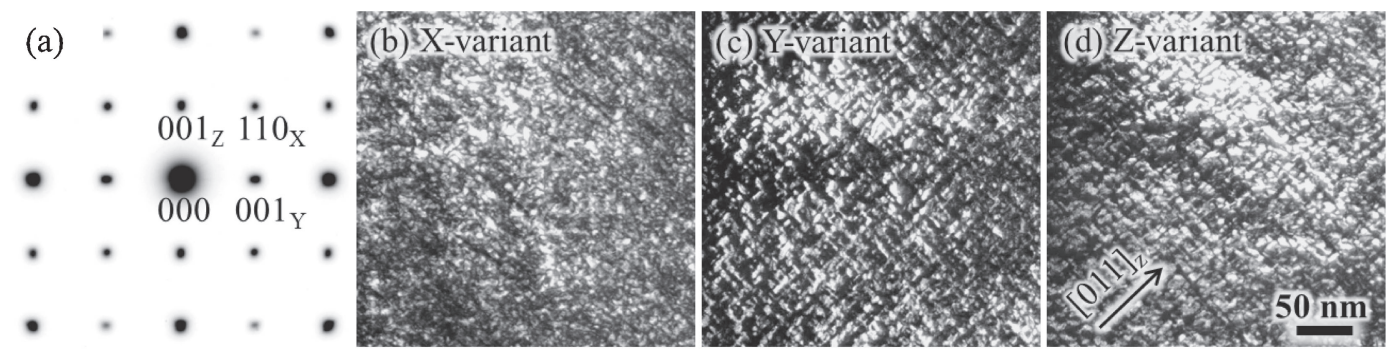

$\mathrm{EB} / /[001]_{\mathrm{X}},[010]_{\mathrm{Y}},[100]_{\mathrm{Z}}$

Fig. 4 (a) SAEDP for specimen $\mathrm{B}$ in Table 1 heat-treated at $773 \mathrm{~K}$ for $30 \mathrm{~min}$ under a magnetic field of $10 \mathrm{~T}$ and $773 \mathrm{~K}$ for 20 min without magnetic field in the first and second steps, respectively. (b)-(d) DFIs of X-, Y- and Z-variants taken by using $110_{\mathrm{X}}, 001_{\mathrm{Y}}$ and $001_{\mathrm{Z}}$ SLRs in (a).

taken from areas $\mathrm{B}$ and $\mathrm{C}$ in (a), respectively. The inverse fast Fourier transformation treatment was applied to all the images. As the contrast of the HAADF-STEM image depends on atomic number, i.e., $Z$-contrast, brighter and darker contrasts correspond to $\operatorname{Pt}(Z=78)$ and $\operatorname{Co}(Z=27)$ atomic columns, respectively. Many lattice fringes corresponding to the $\mathrm{X}$-, Y- and Z-variants are observed. The ordered domain size is about $5 \mathrm{~nm}$ consistent with the DFIs in Fig. 2. Many of interfaces between the Y- and Z-variants are aligned along $\{011\}_{\mathrm{Ll}_{1}}$ with twin relation as shown in Figs. 3(b) and 3(c). This arrangement of two out of three variants is in good agreement with the previous theoretical analysis based on elastic interaction of variant formation associated with $\mathrm{A} 1-\mathrm{L} 1_{0}$ transformation in $\mathrm{Fe}-\mathrm{Pd} .{ }^{12)}$ It is likely that the tweed contrast corresponds to the above twin relation.

Figures 4(a)-4(d) show SAEDP and DFIs after the second step of ordering at $773 \mathrm{~K}$ for $20 \mathrm{~min}$ (specimen B). There is no remarkable difference in SAEDP as well as DFIs compared with Fig. 2, although connection of ordered domains can be observed in Fig. 4(d). This result indicates that twins are introduced and coarsened through the tweed microstructure continuously. To investigate the growth process of preferential variant clearly, the second step of ordering was carried out at $873 \mathrm{~K}$ for $90 \mathrm{~min}$ (specimen C). DFIs in Figs. 5(b) $-5(\mathrm{~d})$ are taken by using $110_{\mathrm{X}}, 001_{\mathrm{Y}}$ and $001_{\mathrm{Z}}$ SLRs in (a). There are also elongation and/or split observed in the SLRs and fundamental reflections in (a), which is probably due to the twinned microstructure as described below. It is determined from the SAEDP, DFIs and trace analysis that $\mathrm{X}-/ \mathrm{Y}-, \mathrm{Y}-/ \mathrm{Z}-$ and $\mathrm{Z}-/ \mathrm{X}$-variants are in twin relation with respect to $\{011\}_{\mathrm{L} 1_{0}}$, although the twin interface between $\mathrm{X}$-/Y- and Z-/X-variants are not edge-on state in Fig. 5. Therefore, we discuss on DFIs in Figs. 5(c) and 5(d) here. It is apparent that the fraction of the Z-variant is higher than that of the Y-variant. These observations indicate that the growth process of preferential Z-variant proceeds by modulation of twins. 

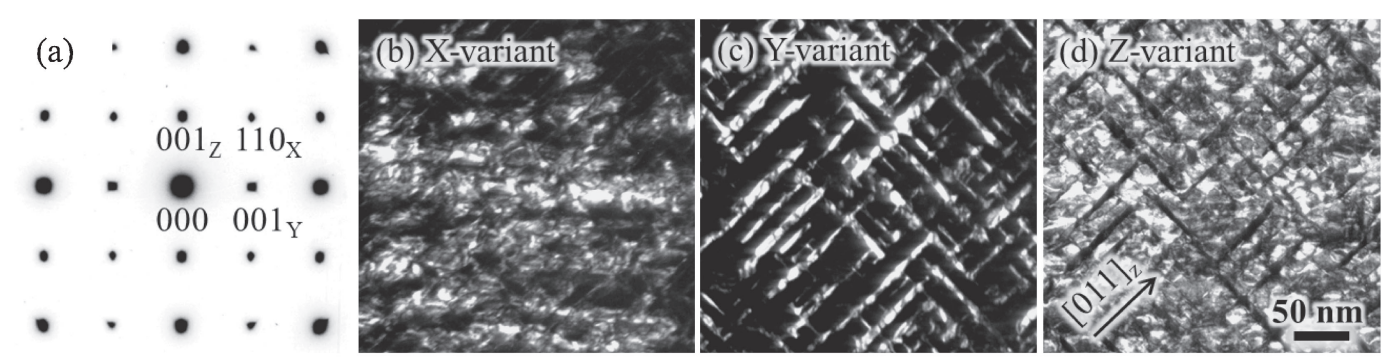

$\mathrm{EB} / /[001]_{\mathrm{X}},[010]_{\mathrm{Y}},[100]_{\mathrm{Z}}$

Fig. 5 (a) SAEDP for specimen $\mathrm{C}$ in Table 1 heat-treated at $773 \mathrm{~K}$ for $30 \mathrm{~min}$ under a magnetic field of $10 \mathrm{~T}$ and $873 \mathrm{~K}$ for 90 min without magnetic field in the first and second steps, respectively. (b)-(d) DFIs of X-, Y- and Z-variants taken by using $110_{\mathrm{X}}, 001_{\mathrm{Y}}$ and $001_{\mathrm{Z}}$ SLRs in (a).

\section{(a)}

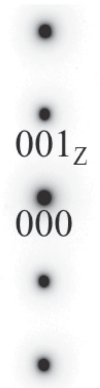

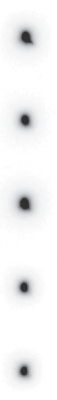

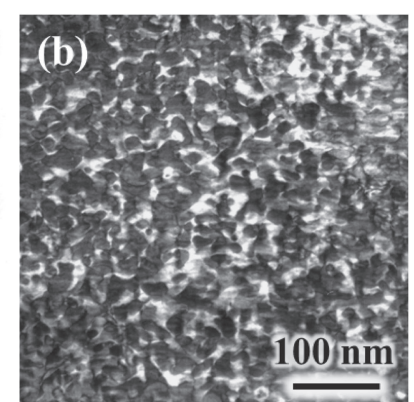

$\mathrm{EB} / /[001]_{\mathrm{X}},[010]_{\mathrm{Y}},[100]_{\mathrm{Z}}$

Fig. 6 (a) SAEDP for specimen $\mathrm{D}$ in Table 1 heat-treated at $773 \mathrm{~K}$ for $30 \mathrm{~min}$ under a magnetic field of $10 \mathrm{~T}$ and $1023 \mathrm{~K}$ for $3 \mathrm{~min}$ without magnetic field in the first and second steps, respectively. (b) Bright field image after formation of single variant.

Figures 6(a) and 6(b) show SAEDP and bright field image after the second step of ordering at $1023 \mathrm{~K}$ for $3 \mathrm{~min}$ (specimen D). As seen in SAEDP, the specimen is in single variant state and only SLR of the Z-variant is observed. The preferential growth of the Z-variant is completed after $3 \mathrm{~min}$ at $1023 \mathrm{~K}$, although there are many of anti-phase domain boundaries.

\section{Conclusions}

Microstructure formation and variant selection during the two-step ordering heat-treatment has been investigated in CoPt. After the first step of ordering under a magnetic field of $10 \mathrm{~T}$ ordered domains of $5 \mathrm{~nm}$ in size were observed by TEM and STEM. The ordered domains tend to align along $\{011\}_{\mathrm{L} 1_{0}}$ to reduce transformation strain. With the progress of ordering micro-twins are formed and fraction of the Z-variant increases by modulation of twins. After the second step of ordering, single variant state is obtained.

\section{Acknowledgments}

The authors are grateful to Prof. S. Nishijima of Osaka University for providing the superconducting magnet through the accomplishment of this work. The authors also thank Dr. M. Itakura of Kyushu University for helpful discussions and comments. One of the authors (H. A.) thanks Ms. Y. Tanaka for assistance with the experiments.

\section{REFERENCES}

1) K. Tanaka, T. Ichitsubo, M. Amano, M. Koiwa and K. Watanabe: Mater. Trans. JIM 41 (2000) 917-922.

2) K. Tanaka, T. Ichitsubo and M. Koiwa: Mater. Sci. Eng. A 312 (2001) 118-127.

3) H. Shima, K. Oikawa, A. Fujita, K. Fukamichi, K. Ishida and A. Sakuma: Phys. Rev. B 70 (2004) 224408.

4) H. Shima, K. Oikawa, A. Fujita, K. Fukamichi and K. Ishida: Appl. Phys. Lett. 86 (2005) 112515.

5) S. Farjami, M. Yasui, T. Fukuda and T. Kakeshita: Scr. Mater. 58 (2008) 811-814.

6) S. Farjami, T. Fukuda and T. Kakeshita: Mater. Trans. 49 (2008) 19701974.

7) S. Farjami, T. Fukuda and T. Kakeshita: ISIJ Int. 49 (2009) 1610-1614.

8) P. Moine, G. M. Michal and R. Sinclair: Acta Metall. 30 (1982) 109123.

9) M. Hirabayashi and S. Weissmann: Acta Metall. 10 (1962) 25-36.

10) J. M. Penisson, A. Bourret and Ph. Eurin: Acta Metall. 19 (1971) 11951204.

11) R. Oshima, M. Sugiyama and F. E. Fujita: Metall. Trans. A 19 (1988) $803-810$.

12) T. Ichitsubo, M. Nakamoto, K. Tanaka and M. Koiwa: Mater. Trans. JIM 39 (1998) 24-30. 